\title{
Population-Based Intervention for Liver Fluke Prevention and Control in Meuang Yang District, Nakhon Ratchasima Province, Thailand
}

\author{
Pontip Kompor' ${ }^{1}$, Rattikarn Muang Karn², Jun Norkaew ${ }^{2}$, Jirawoot Kujapun², \\ Mali Photipim² ${ }^{2}$, Sukanya Ponphimai ${ }^{2}$, Wasugree Chavengkun², Somkiat Phong \\ Paew $^{2}$, Soraya Kaewpitoon ${ }^{3}$, Ratana Rujirakul ${ }^{4}$, Parichart Wakhuwathapong ${ }^{4}$, \\ Tanida Phatisena ${ }^{5}$, Thawatchai Eaksanti ${ }^{5}$, Apinya Joosiri ${ }^{4}$, Poowadol \\ Polsripradistdist $^{6}$, Natnapa Padchasuwan ${ }^{7}$, Natthawut Kaewpitoon ${ }^{8 *}$
}

\begin{abstract}
Opisthorchiasis is still a major health problem in rural communities of Thailand. Infection is associated with cholangiocarcinoma (CCA), which is found frequently in Thailand, particularly in the northeastern. Therefore, this study aimed to evaluate the effectiveness of health intervention in the population at risk for opisthorchiasis and CCA. A quasi-experimental study was conducted in Meuang Yang district, Nakhon Ratchasima province, northeastern Thailand, between June and October 2015. Participants were completed health intervention comprising 4 stations; 1, VDO clip of moving adult worm of liver fluke; 2 , poster of life cycle of liver fluke; 3 , microscopy with adult and egg liver fluke; and 4, brochure with the knowledge of liver fluke containing infection, signs, symptoms, related disease, diagnosis, treatment, prevention, and control. Pre-and-post-test questionnaires were utilized to collect data from all participants. Students paired t-tests were used to analyze differences between before and after participation in the health intervention. Knowledge (mean difference $=-7.48, t=-51.241,95 \% \mathrm{CI}$, $-7.77,-7.19$, p-value $=0.001)$, attitude (mean difference=-9.07, $t=-9.818,95 \% \mathrm{CI}=-10.9,-7.24$, $\mathrm{p}$-value=0.001), and practice (mean difference $=-2.04, t=-2.688,95 \% \mathrm{CI}=-3.55,-0.53$, $\mathrm{p}$-value $=0.008$ ), changed between before and after time points with statistical significance. Community rules were concluded regarding: (1) cooked cyprinoid fish consumption; (2) stop under cooked cyprinoid fish by household cooker; (3) cooked food consumption; (4) hygienic defecation; (5) corrected knowledge campaign close to each household; (6) organizing a village food safety club; (7) and annual health check including stool examination featuring monitoring by village health volunteers and local public health officers. The results indicates that the present health intervention program was effective and easy to understand, with low cost and taking only a short time. Therefore, this program may useful for further work at community and provincial levels for liver fluke prevention and control.
\end{abstract}

Keywords: Population-based intervention - liver fluke - prevention and control - Thailand

Asian Pac J Cancer Prev, 17 (2), 685-689

\section{Introduction}

Opisthorchis viverrini, a carcinogenic liver fluke, is endemic in the Lower Mekong Basin, including Thailand, Lao People's Democratic Republic, Cambodia and central Vietnam (Sripa et al., 2010). The under-estimate of infections are considered, more than 10 million people are infected with $O$. viverrini in Thailand and Lao PDR (Sithithaworn et al., 2012). In Thailand, it is estimated that 6 million people are infected with the $O$. viverrini (Jongsuksuntikul and Imsomboon, 2003). This figure indicated that it is a serious public health problem in Thailand, particularly in northeastern and northern region (Kaewpitoon et al., 2008; Sripa et al., 2010). The $O$. viverrini infection is associated with hepatobiliary diseases including hepatomegaly, cholangitis, cholecystitis, and gallstones (Harinasuta and Vajrasthira 1960; Thamavit et al., 1978; Harinasuta et al., 1984). Recently, $O$.

${ }^{1}$ Master of Public Health, ${ }^{2}$ Faculty of Public Health, Vongchavalitkul University, ${ }^{3}$ School of Family Medicine and Community Medicine, ${ }^{4}$ Parasitic Disease Research Unit, ${ }^{8}$ Suranaree University of Technology Hospital, Suranaree University of Technology, ${ }^{5}$ Faculty of Public health, Nakhon Ratchasima Rajabhat University, ${ }^{6}$ Provincial Public Health Office of Nakhon Ratchasima, Nakhon Ratchasima,${ }^{7}$ Faculty of Public Health, Khon Kaen University, Khon Kaen, Thailand ${ }^{*}$ For correspondence: natthawut.k@hotmail.com 
viverrini has been classified as Type 1 carcinogens by the International Agency for Research on Cancer, World Health Organization (WHO) (IARC, 1994).

The $O$. viverrini infection in Thailand was the first reported in 1955 (Sadun) and many strategies has been operated over period 1955-2000, the national prevalence of $O$. viverrini infection had fallen from $63.6 \%$ to $9.6 \%$ but the high prevalence rate is still found in the rural communities of provinces, Northeast (Sithithaworn et al., 2012). In addition, the high mortality rate of CCA was reported in the northeast areas where found frequently of O. viverrini infection (Sripa et al., 2010). Mortality rate of liver cancer and $O$. viverrini infection rate in different regions of Thailand has been reported and found that Nakhon Ratchasima province has $13.67-16.2$ per 100,000 populations. Eradication of the fluke and identification of high-risk populations are urgently needed (Sripa and pairojkul, 2008). In addition, the distribution $O$. viverrini infection in Nakahon Ratchasima province has been reported, the prevalence of survey in 2009 was $4.6 \%$ (Sitthithaworn et al., 2012). A total of 1,168 stool samples were obtained from 516 males and 652 females. Stool examination showed that $2.48 \%$ were infected with $O$. viverrini (Kaewpitoon et al., 2012c). Furthermore, a total of 640 Cyprinidae family fish including 5 species were collected from different study sites of Nakhon Ratchasima province, and investigated for $O$. viverrini metacercariae. The infection rate was $12.3 \%$ (79/640), predominantly in Cyclocheilichthys armatus, C. repasson, Puntioplites proctzysron, Hampala macrolepitota and Hampala dispar, respectively. The prevalence of $O$. viverrini metaceria was found covered $78.1 \%$ of areas, predominantly in Sida and KiaKham Thale So (Kaewpitoon et al., 2012a).

These figure indicate that $O$. viverrini infection is still a problem in this areas, a population-based approach to behavioral change in highly risk areas are need required. Therefore, aimed to evaluate the effectiveness of health intervention in the population at risk for opisthorchiasis and CCA was conducted in Meuang Yang district, Nakhon Ratchasima province, northeastern Thailand, between June and October 2015. This data is able useful for further work in high risk areas.

\section{Materials and Methods}

\section{Population, sample size, and sampling}

Quasi-experimental study was conducted in Meuang Yang district, Nakhon Ratchasima province, northeastern Thailand, between June and October 2015. The study protocol was approved by Suranaree University Ethical Review Committee, EC58-48. Meuang Yang is a district in the northeastern part of Nakhon Ratchasima province. Neighboring districts are (from the north clockwise) Ban Mai Chaiyapot, Phutthaisong and Kho Mueang of Buriram province, Lam Thamenchai, Chum Phuang and Prathai of Nakhon Ratchasima Province. The district is subdivided into 4 sub-districts, and 44 villages. This district is coverage areas $255.5 \mathrm{~km}^{2}$ (98.6 sq mi), and has 28,359 populations.

Populations at risk for opisthorchiasis and CCA, were screened by using Korat CCA verbal screening test (KCVST) which was contained the history with (1) opisthorchiasis (2) under-cooked fish consumption, (3) praziquantel used (4) cholangitis or cholecystitis, (5) relative family with CCA (6) naive northeastern people, (7) agriculture, and (8) alcohol consumption. Population at risk was identified following $1+2+3+4+5+6+7+8$, who had a score with more than 5 points was selected. Of 100 participants was purposive selected from the calculated score of KCVST who habitat in 4 sub-districts (25 participant/sub-district: included 1 head of village, 1 represent of district administrative organization, 4 village health volunteers, and 19 population at risk who had highest of score).

\section{Measurement and data collection}

Participants were divided into 4 groups (25 participants/ group) and completed interviewed with the pre-test questionnaire. All participants had orientated and each group participated the station of liver fluke knowledge with for 30 minutes and then rotated coverage 4 stations. All station had 1 facilitators for gave them knowledge included; station 1: VDO clip of moving adult worm and story of patient who infected with liver fluke and CCA, station 2: poster of life cycle of liver fluke, station 3: Microscopy with adult and egg of liver fluke, station 4: brochure with the knowledge of liver fluke containing infection, sign, symptom, related disease, diagnosis, treatment, prevention, and control. When participants attended finish all station, each group was brainstorming for liver fluke prevention and control, and then presented their data. Community rules were selected by themselves and conclusion was a final step of process by modulator. All community rules were posted and notice in each village. Local public health officer and village health volunteer, who had been trained for monitoring, went to home visit all participant for a month, 1 time/week. Posttest was asked each participant after health intervention 1 month later by local public health officers (Figure 1,2, 3). Pre-and-post-test; a predesigned KAP questionnaire

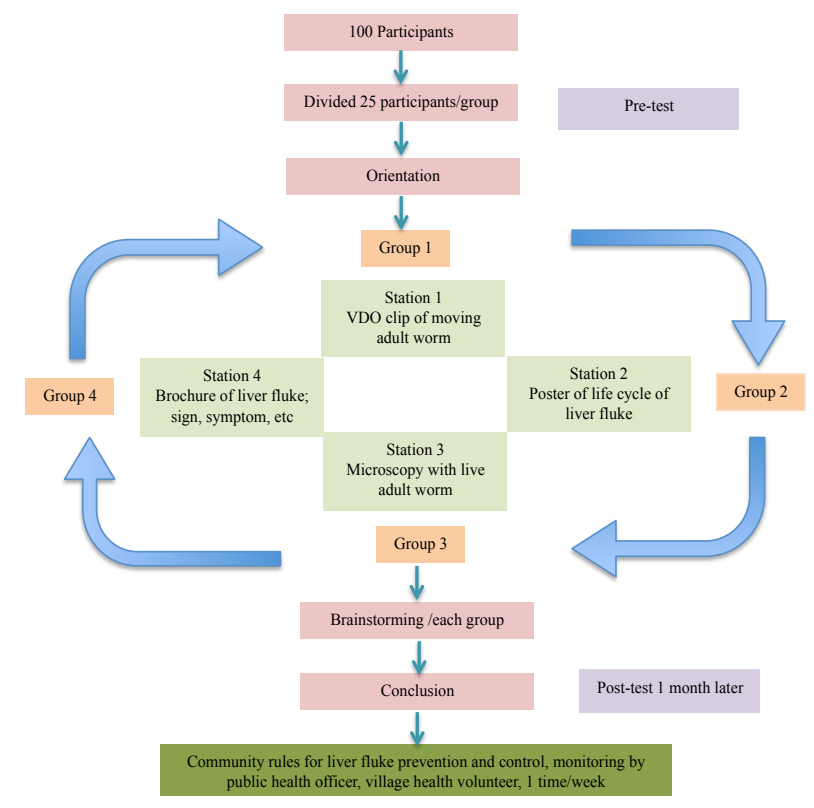

Figure 1. Diagram of Health Intervention for Participants 
Population-Based Intervention for Liver Fluke Prevention and Control in Nakhon Ratchasima Province, Thailand was utilized to collect the data from all participants. The questionnaire was comprised 5 domain included (1) demographic data; gender, age group, education, agriculture, (2) past history with liver fluke and perception, (3) knowledge; 15 questions, (4) attitude; 20 questions, and (5) practice; 20 questions. Reliability and validity of questionnaire was analyzed, knowledge (KruderRichardon-20) $=0.85$, attitude and practice (coefficient of Cronbach's alpha) $=0.92$ and 0.76 , respectively.

\section{Statistical analysis}

Descriptive and analytical statistical data were analyzed with SPSS software. Each questionnaire was analyzed and interpreted for their parts. Evaluation of knowledge level was calculated and analyzed according to Bloom et al (1971), answer correct=1, incorrect=0, and interpreted to high level; $11-15$ points, moderate level; 6-10 points, 0-5 points; low level. Evaluation of attitude and practice level was calculated and analyzed, attitude with 3 choice (agree, not sure, dis-agree): positive question $=3,2,1$, negative question $=1,2,3$, and interpreted to good level; 48-60 points, moderate level; 36-47 points, and low level; 0-35 points, practice with 3 choice (frequently, sometimes, never): positive question=3,2,1, negative question=1,2,3, and interpreted to good level; 48-60 points, moderate level; 36-47 points, poor level; 0-35 points. Student pair-t-testing was used to analyze the difference between before and after participated the health intervention.

\section{Results}

A total of 100 participants was included in this study. The majorities of participants were female (73.00\%), age group 46-55 years old $(48.00 \%)$, primary school $(92.00 \%)$, marriage $(91.00 \%)$, agriculture $(99.00 \%)$, income 5,001-15,000 bah/month (36.00\%) (Table 1). Participants had received information about liver fluke

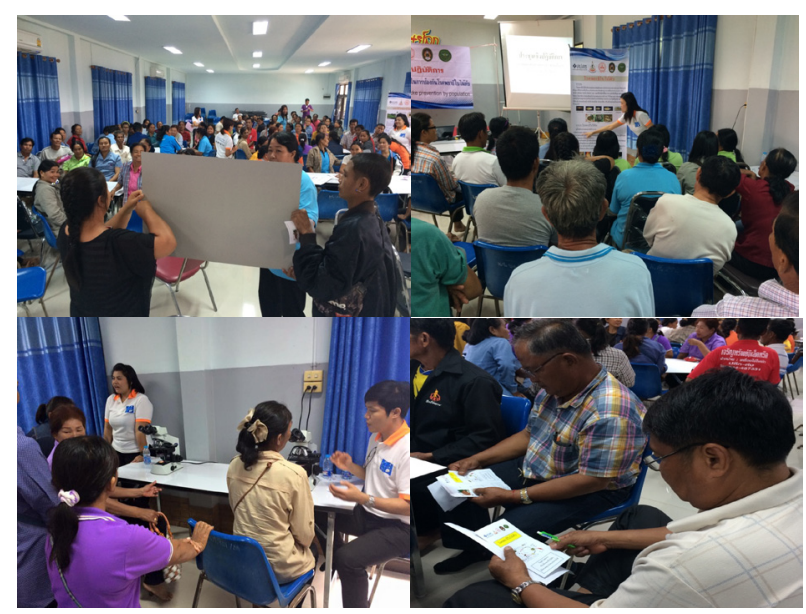

Figure 2. Group Process and Health Intervention Program Through VDO Clip of Moving Adult Worm and Story of Patient who Infected with Liver Fluke and CCA, Poster Presentation of Life Cycle of Liver Fluke, Demonstration of adult Worm and Egg of Liver Fluke Using Microscopy, and Brochure of liver Fluke Information from many resource, the majorities were public health officer $(72.00 \%)$, followed by village health volunteer $(64.00 \%)$, brochure $(53.00 \%)$, and news station of village (30.00\%) (Table 2).

The histories about examination, infection, and treatment of liver fluke, and CCA, were interviewed and found that participants had ever stool examined $33.00 \%$, liver fluke infection $21.00 \%$, praziquantel used $76.00 \%$, period of praziquantel used within $1-2$ years $62.00 \%$, and relative with CCA $3.00 \%$ (Table 3)

Participant had low knowledge in the group of before intervention (mean=3.79, $\mathrm{SD}=0.74$ ), and the high level was found in this group after intervention

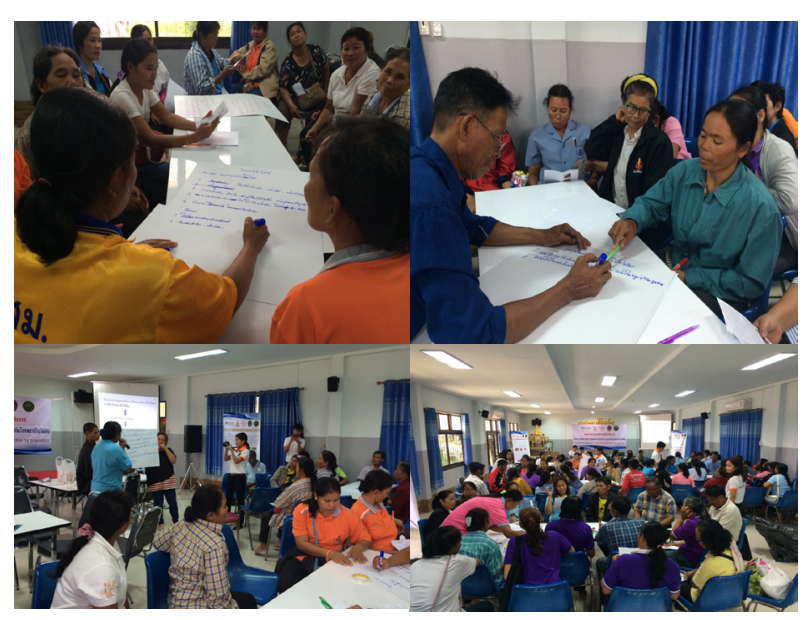

Figure 3. Brain Storming and Public Hearing for Community Rules of Liver Fluke Prevention and in Their Villages

Table 1. Characteristic baseline of participant the health behavior intervention in Meuang Yang district, Nakhon Ratchasima province, Thailand

\begin{tabular}{|c|c|c|}
\hline Characteristic baseline & No. of participant & $\%$ \\
\hline \multicolumn{3}{|l|}{ Gender } \\
\hline Male & 27 & 27.00 \\
\hline Female & 73 & 73.00 \\
\hline \multicolumn{3}{|l|}{ Age } \\
\hline $25-35$ & 3 & 3.00 \\
\hline $36-45$ & 31 & 31.00 \\
\hline $46-55$ & 48 & 48.00 \\
\hline $56-65$ & 16 & 16.00 \\
\hline$>65$ & 2 & 2.00 \\
\hline \multicolumn{3}{|l|}{ Education } \\
\hline Primary school & 8 & 8.00 \\
\hline Secondary school & 92 & 92.00 \\
\hline \multicolumn{3}{|l|}{ Marital status } \\
\hline Single & 3 & 3.00 \\
\hline Marriage & 91 & 91.00 \\
\hline Widow/separated & 6 & 6.00 \\
\hline \multicolumn{3}{|l|}{ Occupation } \\
\hline Agriculture & 99 & 99.00 \\
\hline Trader & 1 & 1.00 \\
\hline \multicolumn{3}{|l|}{ Income (Baht/month) } \\
\hline$<5,000$ & 13 & 13.00 \\
\hline $5,001-15,000$ & 36 & 36.00 \\
\hline $15,001-25,000$ & 4 & 4.00 \\
\hline $25,001-35,000$ & 21 & 21.00 \\
\hline $35,001-45,000$ & 18 & 18.00 \\
\hline$>45,001$ & 8 & 8.00 \\
\hline
\end{tabular}


(mean=11.27, $\mathrm{SD}=1.22$ ). Participant had a high knowledge after participated the health intervention with the statistical significant (mean difference $=-7.48, \mathrm{t}=-51.241$, $\mathrm{p}$-value $=0.001)$. Participants had a low attitude in before (mean=39.45, SD=7.31) and moderate attitude after (mean=48.52, SD=7.94) participated the health intervention, however, the comparison was analyzed and found that in the both of before and after intervention had a different point with a statistical significant (mean difference $=-9.07, \mathrm{t}=-9.818, \mathrm{p}$-value $=0.001)$. While, Participants had a low practice in before (mean $=38.64$, $\mathrm{SD}=6.95)$ and moderate practice (mean $=40.68, \mathrm{SD}=4.00$ ) participated the health intervention, the comparison was found that they had a different point with a statistical significant (mean difference $=-2.04, \mathrm{t}=-2.688$, p-value $=0.008)$ (Table 4).

Community rules were selected and concluded by all participants, including; (1) cooked cyprinoid fish

Table 2. Information Resources for Participant Regarding to Liver Fluke

\begin{tabular}{lcc}
\hline Resources & Frequencies & $\%$ \\
\hline Head village & 27 & 27.00 \\
Village health volunteer & 64 & 64.00 \\
Public health officer & 72 & 72.00 \\
Neighbor & 1 & 1.00 \\
Nurse & 7 & 7.00 \\
Medical doctor & 1 & 1.00 \\
News station of village & 30 & 30.00 \\
Brochure & 53 & 53.00 \\
Internet & 1 & 1.00 \\
\hline
\end{tabular}

Table 3. Histories of Participant Regarding Stool Examination, Infection, Treatment, and Cholangiocarcinoma

\begin{tabular}{lcc}
\hline Histories & No. of participant & $\%$ \\
\hline Stool examination & & \\
$\quad$ Yes & 33 & 33.00 \\
$\quad$ No & 67 & 67.00 \\
Liver fluke infection & & \\
$\quad$ Yes & 21 & 21.00 \\
$\quad$ No & 79 & 79.00 \\
Praziquantel used & 76 & 76.00 \\
$\quad$ Yes & 24 & 24.00 \\
$\quad$ No & 12 & 12.00 \\
Period of praziquantel used & 62 & 62.00 \\
$\quad$ 6-12 months & 26 & 26.00 \\
1-2 years & & \\
3-4 years & 3 & 3.00 \\
Relative with cholangiocarcinoma & 97.00 \\
$\quad$ Yes & 97 & \\
$\quad$ No & & \\
\hline
\end{tabular}

consumption, (2) stop under cooked cyprinoid fish by household cooker, (3) cooked food consumption, (4) hygienic defecation, (5) corrected knowledge campaign is close up to each household, (6) organizing the village food safety club, (7) annual health check including stool examination in which monitoring by village health volunteer and local public health officer.

\section{Discussion}

Recent study, participants were screened the population at risk for opisthorchiasis and CCA by using verbal screening test, and then we collected the participant who had the highest of score from each sub-districts, including the head of villages, representative district administrative organization, and village health volunteer. The majorities of participants were female, age group 46-55 years old, primary school, marriage, agriculture, had income 5,001$15,000 \mathrm{bah} / \mathrm{month}$. Participants had received information regarding liver fluke from many resources, the majorities were public health officer, followed by village health volunteer, brochure, and news station of village. This figure indicated that public health officer and village health volunteer are the main resources for spread the information of campaign that we used for our project via both announcement and coordination. These key persons were the main resources for contact, activities work, and monitoring of project, therefore, improvement of their knowledge about liver fluke have done. Past histories of participants had ever stool examined $33.00 \%$, had the liver fluke infection $21.00 \%$, praziquantel used $76.00 \%$, and relative with CCA $3.00 \%$. in addition, a low level of knowledge, and moderate levels of attitude and practice regarding liver fluke prevention and control in the participant, was investigated. Previous study indicated that knowledge, attitude, and practice related to liver fluke infection in the northeastern Thailand (Kaewpitoon et al., 2007). This recent result suggest that opisthorchiasis is still a problem in this areas. Therefore, health intervention in the population at risk is need required.

Our health intervention program is designed from basis knowledge regarding liver fluke prevention and control, and has been conducted for 4 month. Lawa model (Sripa et al., 2015); the success model for liver fluke prevention and control in the rural community, this is our inspiration for intervention. In the concept, the project's primary challenge has been learning how to build an effective disease prevention and control program from the "bottom-up", including creating an understanding of liver fluke infection disease risk with the community. Preliminary results suggest treatment and education campaigns are only effective to the extent engage with the

Table 4. Behavioral Levels of Participants who Participated Health Intervention

\begin{tabular}{|c|c|c|c|c|c|c|c|c|c|c|c|}
\hline \multirow[t]{3}{*}{ Behavior } & \multicolumn{6}{|c|}{ Intervention } & \multirow{2}{*}{\multicolumn{3}{|c|}{ Mean difference }} & \multirow[t]{3}{*}{$\mathrm{t}$} & \multirow[t]{3}{*}{ p-value } \\
\hline & \multicolumn{3}{|c|}{ Before intervention } & \multicolumn{3}{|c|}{ After intervention } & & & & & \\
\hline & Mean & SD & Level & Mean & SD & Level & & Lower & Upper & & \\
\hline Knowledge & 3.79 & 0.74 & Low & 11.27 & 1.22 & High & -7.48 & -7.77 & -7.19 & -51.241 & 0.001 \\
\hline Attitude & 39.45 & 7.31 & Low & 48.52 & 7.94 & High & -9.07 & -10.9 & -7.24 & -9.818 & 0.001 \\
\hline Practice & 38.64 & 6.95 & Low & 40.68 & 4.00 & Moderate & -2.04 & -3.55 & -0.53 & -2.688 & 0.008 \\
\hline
\end{tabular}


community in a reciprocal learning process. This includes understanding the local culture and broader health and well-being concerns held by a community and ensuring these are aligned with intervention program approaches and activities. From this data, we build up our preparation and designated a program for study community. Our health intervention comprised station 1: VDO clip of moving adult worm of liver fluke, station 2: poster of life cycle of liver fluke, station 3: Microscopy with adult and egg liver fluke, station 4: brochure with the knowledge of liver fluke containing infection, sign, symptom, related disease, diagnosis, treatment, prevention, and control. Participants had high level of knowledge, high attitude and moderate practice after participated the intervention. This figure suggested that program appropriate for rural people and may useful for liver fluke prevention and control.

Participants had selected and concluded their community rules including; (1) cooked cyprinoid fish consumption, (2) stop under cooked cyprinoid fish by household cooker, (3) cooked food consumption, (4) hygienic defecation, (5) corrected knowledge campaign is close up to each household, (6) organizing the village food safety club, (7) annual health check including stool examination in which monitoring by village health volunteer and local public health officer. This participated community rules is decided to practice in their community and will be further monitoring by village health officers and local public health officers. Certainly, we further visit them again 3, 6 month, and 1 year later for investigate the sustainable rules and the re-infection of liver fluke.

This result indicates that health intervention program has more effectiveness with easily to understand, low cost, short time. Therefore, this program may useful for further work in community and provincial scales for liver fluke prevention and control.

\section{Acknowledgements}

This health intervention program was supported by National Health Security Office of Nakhon Ratchasima province, through health promotion and prevention fund, year 2015. Special thanks all staffs of district public health organization of Meuang Yang for their assistance

\section{References}

Bloom BS (1971). Handbook on formative and summative of student learning. New York: Mc Graw-Hill Book Company.

Harinasuta C, Vajrasthira S (1960). Opisthorchiasis in Thailand. Am J Trop Med Hyg, 54, 100-5.

Harinasuta T, Riganti M, Bunnag D (1984). Opisthorchis viverrini infection: pathogenesis and clinical features. Arzneimittelforschung, 34, 1167-9.

IARC. (1994). Infection with liver flukes (Opisthorchis viverrini, Opisthorchis felineus and clonrochis sinensis). IARC Monogr Eval Carcinog Risks of Hum, 61, 121-75.

Jongsuksuntigul P, Imsomboon T (2003). Opisthorchiasis control in Thailand. Acta Trop, 88, 229-32.

Kaewpitoon N, Kaewpitoon SJ, Pengsaa P, et al (2007). Knowledge, attitude and practice related to liver fluke infection in northeast Thailand. World J Gastroenterol, 13, $1837-40$.
Kaewpitoon N, Kaewpitoon SJ, Pengsaa P(2008). Opisthorchiasis in Thailand: review and current status. World J Gastroenterol, 14, 2297-302.

Kaewpitoon N, Kaewpitoon SJ, Ueng-arporn N, et al (2012). Carcinogenic human liver fluke: current status of Opisthorchis viverrini metacercariae in Nakhon Ratchasima, Thailand. Asian Pac J Cancer Prev, 13, 1235-40.

Kaewpitoon SJ, Rujirakul R, Ueng-Arporn N, et al (2012). Community-based cross-sectional study of carcinogenic human liver fluke in elderly from Surin province, Thailand. Asian Pac J Cancer Prev, 13, 4285-8.

Kaewpitoon SJ, Rujirakul R, Kaewpitoon N (2012). Prevalence of Opisthorchis viverrini infection in Nakhon Ratchasima province, Northeast Thailand. Asian Pac J Cancer Prev, 13, 5245-9.

Sadun EH (1995). Studies on Opisthorchis viverrini in Thailand. Am J Hyg, 62, 81-115.

Sithithaworn P, Andrews RH, Nguyen VD, et al (2012). The current status of opisthorchiasis and clonorchiasis in the Mekong Basin. Parasitol International, 61, 10-6.

Sripa B, Kaewkes S, Intapan PM, et al (2010). Food-borne trematodiases in Southeast Asia: epidemiology, pathology, clinical manifestation and control. Adv Parasitol, 72, 305-50.

Sripa B, Brindley PJ, Mulvenna J, et al (2012). The tumorigenic liver fluke Opisthorchis viverrini-multiple pathways to cancer. Trends Parasitol, 28, 395-407.

Sripa B, Pairojkul C (2008). Cholangiocarcinoma: lessons from Thailand. Curr Opin Gastroenterol, 24, 349-56.

Sripa B, Tangkawattana S, Laha T, et al (2015). Toward integrated opisthorchiasis control in northeast Thailand: The Lawa project. Acta Tropica, 141, 361-7

Thamavit W, Bhamarapravati N, Sahaphong S, et al (1978).Effects of dimethylnitrosamine on induction of cholangiocarcinoma in Opisthorchis viverrini-infected Syrian golden hamsters. Cancer Res, 38, 4634-9. 